\title{
Reduced Lorenz models for anomalous transport and profile resilience
}

\author{
Rypdal, K.; Garcia, Odd Erik
}

Published in:

Physics of Plasmas

Link to article, DOI:

10.1063/1.2435318

Publication date:

2007

Document Version

Publisher's PDF, also known as Version of record

Link back to DTU Orbit

Citation (APA):

Rypdal, K., \& Garcia, O. E. (2007). Reduced Lorenz models for anomalous transport and profile resilience. Physics of Plasmas, 14(2), 022101. https://doi.org/10.1063/1.2435318

\section{General rights}

Copyright and moral rights for the publications made accessible in the public portal are retained by the authors and/or other copyright owners and it is a condition of accessing publications that users recognise and abide by the legal requirements associated with these rights.

- Users may download and print one copy of any publication from the public portal for the purpose of private study or research.

- You may not further distribute the material or use it for any profit-making activity or commercial gain

- You may freely distribute the URL identifying the publication in the public portal

If you believe that this document breaches copyright please contact us providing details, and we will remove access to the work immediately and investigate your claim 


\title{
Reduced Lorenz models for anomalous transport and profile resilience
}

\author{
K. Rypdal \\ Department of Physics and Technology, University of Troms $\phi$, N-9037 Troms $\phi$, Norway \\ O. E. Garcia \\ Association EURATOM-Risф National Laboratory, OPL-128 Risø, DK-4000 Roskilde, Denmark
}

(Received 9 November 2006; accepted 26 December 2006; published online 2 February 2007)

\begin{abstract}
The physical basis for the Lorenz equations for convective cells in stratified fluids, and for magnetized plasmas imbedded in curved magnetic fields, are reexamined with emphasis on anomalous transport. It is shown that the Galerkin truncation leading to the Lorenz equations for the closed boundary problem is incompatible with finite fluxes through the system in the limit of vanishing diffusion. An alternative formulation leading to the Lorenz equations is proposed, invoking open boundaries and the notion of convective streamers and their back-reaction on the profile gradient, giving rise to resilience of the profile. Particular emphasis is put on the diffusionless limit, where these equations reduce to a simple dynamical system depending only on one single forcing parameter. This model is studied numerically, stressing experimentally observable signatures, and some of the perils of dimension-reducing approximations are discussed.

(C) 2007 American Institute of Physics. [DOI: 10.1063/1.2435318]
\end{abstract}

\section{INTRODUCTION}

In plasma physics, the notion of anomalous transport is used indiscriminately to characterize phenomena giving rise to transport coefficients that exceed the collisional diffusive transport by orders of magnitude. Such transport is caused by the excitation of collective motions driven by gradients in thermodynamic quantities such as plasma density and pressure. Similar processes arise in geophysical fluids such as oceans and atmospheres and explain rapid transport of particles and energy, which cannot be explained by collisional diffusion alone. The archetype of an anomalous transport mechanism is the Rayleigh-Bénard (RB) instability and associated thermal convection, driven by a vertical temperature gradient in a fluid stratified by gravity. This instability was first studied experimentally by Bénard in the late 1800s and theoretically by Lord Rayleigh in 1916. ${ }^{1}$ In 1963, Lorenz presented an autonomous set of three coupled ordinary differential equations (a dynamical system) to describe the nonlinear evolution of this instability, and found the deterministic, but unpredictable, aperiodic flows that in modern terminology are called chaos. ${ }^{2}$

The classical mathematical description of the RB instability treats a boundary value problem with fixed temperature and no vertical flow at two given vertical levels. In Sec. II of this paper, we present a brief review of the RB and Lorenz models and demonstrate that the latter model for the thermal convection problem cannot give transport through the fluid layer that greatly exceeds the heat flux due to thermal conduction (collisional diffusion) driven by the imposed temperature difference between the two levels. This observation indicates that the Lorenz model for the convection problem is not suitable to describe situations in which convection enhances the diffusive transport by orders of magnitude. ${ }^{3}$

It has been pointed out by several authors that the RB instability has its counterpart in flute interchange instabilities in magnetized plasmas, driven unstable by pressure gradients in the direction of the radius of magnetic-field curvature. ${ }^{4-10}$ In Sec. III, we explore this analogy in detail by deriving the $\mathrm{RB}$ and the Lorenz models for a particular interchange instability, and discussing the similarities and differences in the underlying physics.

One restriction of the Lorenz truncation for the thermal convection problem becomes particularly apparent in the limit of vanishing heat diffusivity. This limit is discussed in Sec. IV, where the Lorenz equations are cast into a form in which the diffusivity appears explicitly as coefficients in the equations, and thus makes the transition to the diffusionless limit trivial by setting these coefficients to zero. In this section, it is shown explicitly that in the collisionless limit the energy flux through the layer vanishes unless the driving temperature difference goes to infinity. This feature of the classical RB convection problem provides a strong motivation for seeking an alternative formulation that allows for finite fluxes, even in the diffusionless limit. Focusing on the plasma transport issue, we therefore proceed in Sec. V to formulate a model for interchange motions and plasma transport by relaxing the boundary conditions that do not allow convective transport through the boundaries. We name this formulation the convective streamer model, as opposed to the traditional convective cell model.

In Sec. VI, we present results of a numerical study of the diffusionless Lorenz equations (DLE), with emphasis on the features that have the potential to be observable experimentally. We contrast these solutions with those of the full Lorenz equations (LE) with very small diffusivity. Some qualitative features survive the transition from the full to the reduced model, while others do not. In Sec. VII, we study this structural instability in some detail, and also the structural stability of introducing a further reduction of the DLE, the so-called slaved model, which might be justified in a certain parameter range. This discussion is intended to illustrate and elucidate aspects of an extremely important prob- 
lem in nonlinear science, which is the degree to which physical reality can be described by low-dimensional models whose dynamics may not be robust with respect to addition or removal of small physical effects. In Sec. VIII, we summarize our findings and discuss how the low-dimensional dynamics predicted by these models can be detected in real plasma systems.

\section{THE LORENZ MODEL FOR THERMAL CONVECTION}

The two-dimensional RB convection model describes the motion of a fluid sandwiched between two horizontal plates separated by a distance $d$ and subject to a temperature difference $\Delta T$. Heated from below, the fluid close to the lower plate is subject to expansion, and when the temperature gradient exceeds a threshold that renders a positive energy gain by interchanging heavy fluid on top with light fluid below, taking into account losses due to viscosity and heat conduction, the RB instability develops, creating convection rolls of horizontal size that minimize the threshold of the instability. From the continuity equation, the momentum equation, the heat conduction equation, and a linear law for heat expansion of the fluid, one can easily derive the two coupled equations for the normalized stream function $\psi(x, y, t)$ and the normalized temperature deviation $\theta(x, y, t)$ from a linear vertical temperature profile, ${ }^{10}$

$$
\begin{aligned}
& \left(\frac{\partial}{\partial t}+\hat{\mathbf{z}} \times \nabla \psi \cdot \nabla\right) \nabla_{\perp}^{2} \psi+\operatorname{PrRa} \frac{\partial \theta}{\partial y}=\operatorname{Pr}_{\perp}^{4} \psi, \\
& \left(\frac{\partial}{\partial t}+\hat{\mathbf{z}} \times \nabla \psi \cdot \nabla\right) \theta+\frac{\partial \psi}{\partial y}=\nabla_{\perp}^{2} \theta .
\end{aligned}
$$

Here a rectangular coordinate system is employed with $x$ in the vertical direction, and $y$ and $z$ in horizontal directions with $z$ along the axes of the convection rolls. The variables have been made dimensionless through the substitutions

$$
t \rightarrow \frac{\chi}{d^{2}} t, \quad \mathbf{x} \rightarrow \frac{\mathbf{x}}{d}, \quad \psi \rightarrow \frac{\psi}{\chi}, \quad \theta=\frac{T-T_{0}(x)}{\Delta T},
$$

where the dimensionless velocity is given by the dimensionless stream function through $\hat{\mathbf{z}} \times \nabla \psi$ and $T_{0}(x)=T_{0}(0)$ $-(x / d) \Delta T$ is the linear temperature profile that prevails below instability threshold. The dimensionless control parameters are the Prandtl and Rayleigh numbers defined, respectively, by

$$
\operatorname{Pr}=\frac{\mu}{\chi}, \quad \operatorname{Ra}=\frac{\alpha g d^{3} \Delta T}{\mu \chi},
$$

where $\mu$ is the kinematic viscosity, $\chi$ is the thermal diffusivity, $\alpha$ is the thermal expansion coefficient, and $g$ is the gravitational acceleration.

Lorenz made a particularly simple analysis of thermal convection close to the instability threshold by means of a modal truncation given by

$$
\psi(x, y, t)=\mathcal{A}(t) \sin (K y) \sin (\pi x),
$$

$$
\theta(x, y, t)=\mathcal{B}(t) \cos (K y) \sin (\pi x)-\mathcal{C}(t) \sin (2 \pi x),
$$

where in the nondimensional units the vertical and horizontal extension of one convection roll is unity and $\pi / K$, respectively. The truncation above satisfies boundary conditions corresponding to free-slip and stress-free, and constant temperature on the boundaries. The modal truncation (4) satisfies Eq. (1a) exactly, but the nonlinear version of Eq. (1b) can only be approximately satisfied. The terms proportional to $\mathcal{A}(t)$ and $\mathcal{B}(t)$ represent the convection rolls, whereas the term proportional to $\mathcal{C}(t)$ represents a modification of the temperature profile induced by this nonlinearity. Insertion of Eqs. (4) into Eqs. (1), and neglecting an uncompensated term of the form $\sin (3 \pi x)$, known as a modal or Galerkin truncation, yields the well-known Lorenz equations, ${ }^{2}$

$$
\begin{aligned}
& \dot{\mathcal{X}}=\sigma(\mathcal{Y}-\mathcal{X}), \\
& \dot{\mathcal{Y}}=r \mathcal{X}-\mathcal{X Z}-\mathcal{Y}, \\
& \dot{\mathcal{Z}}=\mathcal{X} \mathcal{Y}-b \mathcal{Z} .
\end{aligned}
$$

These equations have been brought into the standard form used in the chaos literature by means of the following redefinition of variables:

$$
\begin{aligned}
& \mathcal{X}(t)=\frac{\pi K}{\left(\pi^{2}+K^{2}\right) \sqrt{2}} \mathcal{A}(t), \quad \mathcal{Y}(t)=\frac{\pi r}{\sqrt{2}} \mathcal{B}(t), \\
& \mathcal{Z}(t)=\pi r \mathcal{C}(t),
\end{aligned}
$$

and the control parameters,

$$
\sigma=\operatorname{Pr}, \quad r=\frac{K^{2}}{\left(\pi^{2}+K^{2}\right)^{3}} \operatorname{Ra}, \quad b=\frac{4 \pi^{2}}{\pi^{2}+K^{2}} .
$$

One should note that the convection model (1) and the Lorenz equations (5) describe the evolution of perturbations around the hydrostatic, diffusive equilibrium of the original momentum and heat conduction equations. This equilibrium corresponds to the fixed point $\mathcal{X}=\mathcal{Y}=\mathcal{Z}=0$ of the Lorenz equations. Stability of this equilibrium is investigated by linearization, which decouples the profile equation $(5 c)$ from Eqs. (5a) and (5b) for the convection rolls. Since $b>0$, the temperature profile relaxes exponentially to the equilibrium profile $(\mathcal{Z} \rightarrow 0)$, so we only need to investigate the linear stability of Eqs. (5a) and (5b), which trivially yields instability if $r>1$, corresponding to $\mathrm{Ra}>K^{2} /\left(\pi^{2}+K^{2}\right)^{3}$. The minimum Rayleigh number giving rise to instability occurs when $K=\pi / \sqrt{2}$, thus the most unstable convection cells are the ones with aspect ratio $\pi / K=\sqrt{2}$. The corresponding critical Rayleigh number is $\mathrm{Ra}_{c}=27 \pi^{4} / 4$, and the parameter $b$ for this aspect ratio is $b=8 / 3$. In many studies of the Lorenz system, $b$ is fixed at this value. This makes sense for the RB convection problem, but not necessarily for the plasma applications dealt with in this paper, where $K$ may be determined by other geometric constraints and boundary conditions. 
From the definition of Ra, we observe that the condition $\mathrm{Ra}>\mathrm{Ra}_{c}$ imposes a threshold condition on the temperature gradient,

$$
\Delta T>\Delta T_{c}=\frac{\mathrm{Ra}_{c}}{\alpha g d^{3}} \mu \chi .
$$

Note that this threshold depends crucially on the dissipation coefficients. In the limit of vanishing dissipation, an arbitrarily weak inverse stratification leads to instability. When the dissipative equilibrium is unstable, that is, for $r>1$, there exist two other fixed points in addition to the trivial one, given by $\mathcal{X}=\mathcal{Y}= \pm \sqrt{b(r-1)}$ and $\mathcal{Z}=r-1$. For $b=8 / 3$ and $1<r<13.93$ these fixed points are stable and represent states of steady convection. Such states also exist for 13.93 $<r<24.06$, but for initial conditions outside small basins of attraction around these fixed points, the phase-space trajectories are transient chaotic oscillations around the fixed points with amplitudes of order $r$. For $r>24.06$, the transient chaos is converted to a chaotic attractor (a crisis), and for $r>24.74$ the fixed points become unstable (a Hopf bifurcation), and all trajectories are either limit cycles or chaotic with amplitudes of order $r$.

The stable fixed points represent steady convection, where heat is transported also by convection inside the fluid layer, but solely by conduction through the boundaries. The heat flux through the fluid layer is in dimensional units given by

$$
\begin{aligned}
\Gamma=-\left.\chi \frac{\partial T}{\partial x}\right|_{x=0} & =\chi \Delta T\left[\frac{1}{d}+\mathcal{C} \frac{\partial}{\partial x} \sin \left(\frac{2 \pi x}{d}\right)\right]_{x=0} \\
& =\frac{\chi \Delta T}{d}(1+2 \pi \mathcal{C}) .
\end{aligned}
$$

Using the definition of $\mathcal{Z}=\pi r \mathcal{C}$, and the solution for the nontrivial fixed point, $\mathcal{Z}=r-1$, Eq. (9) reduces to

$$
\Gamma=\frac{\chi \Delta T}{d}\left(3-\frac{2}{r}\right) .
$$

This means that, for the fixed points of the Lorenz model, the heat flux will be limited to three times the diffusive flux driven by the linear temperature profile of the basic state, even in the limit of very strong drive, that is, large $r$. Moreover, when the fixed points are unstable for large $r$, the amplitudes of the convection rolls and the profile modification oscillate periodically or chaotically. But since we always have $\mathcal{Z} \sim r$, the heat flux in the Lorenz truncation will not greatly exceed $3 \chi \Delta T / d$. For large Rayleigh numbers, the Lorenz truncation fails to properly describe the convective motions, and the heat flux can increase above the conductive level of the basic state by orders of magnitude. ${ }^{9,10}$ The strongly restricted heat flux allowed by the Lorenz model for the RB problem is a severe limitation of the low dimensional model, which we will remove in Sec. V.

\section{THE LORENZ MODEL FOR PLASMA CONVECTION CELLS}

We now turn to the problem of the pressure gradient driven instability of a plasma immersed in a curved un- sheared magnetic field. The simplest model for such a magnetic field is the solenoidal field $\mathbf{B}=-\left(B_{0} R_{0} / R\right) \hat{\boldsymbol{\Phi}}$ generated by an electric current flowing along the positive $Z$ axis in a cylindrical coordinate system $(R, \Phi, Z)$. If the plasma is bounded in the axial direction, it is easily shown that a simple magnetohydrodynamic equilibrium does not exist. But a collisionless plasma that is infinite and uniform in the $Z$ direction is in equilibrium irrespective of the shape of the pressure profile. It can also be shown that equilibrium of an axially uniform plasma of finite axial extension exists if it is bounded by conducting walls. ${ }^{11}$ However, such an equilibrium is subject to interchange instability of field-aligned filaments, where the driving mechanism has many similarities to the RB instability. The field curvature gives rise to an effective gravity pointing radially outwards, and pressure gradients will drive interchange motions. There are, however, also some important differences. One which we will discuss below is that the interchange instability exhibits a nontrivial pressure gradient threshold that prevails even in the absence of dissipation. This threshold is associated with the compressible nature of $\mathbf{E} \times \mathbf{B}$ motions of fluid elements in a nonuniform magnetic field. ${ }^{12-14}$

We shall illustrate the basic features of interchange dynamics by analyzing a model for a low-beta plasma where the electrostatic approximation can be invoked. For the sake of simplicity, we will also assume isothermal electrons, thus illustrating that temperature gradients are not necessary to drive interchange instability. We note, however, that nonisothermal models give rise to similar model equations. Consider a solenoidal magnetic field as described above, and a cylindrical plasma shell of thickness $d$ centered around the radius $R_{0}$. We shall again assume the thin layer approximation, implying that $d \ll R_{0}$. In the isothermal approximation, the fundamental plasma variables are the electric potential $\phi$ and the electron number density $n$. In order to make a connection to the fluid convection problem, we introduce the stream function $\psi=\phi / B_{0}$. Moreover, it is conventional to introduce the logarithm of electron number density $N=\ln n$. From the momentum and continuity equations, taking into account the effects of field curvature, we then obtain the vorticity and particle number conservation equations in the form $^{11,12}$

$$
\begin{aligned}
& \left(\frac{\partial}{\partial t}+\hat{\mathbf{z}} \times \nabla \psi \cdot \nabla\right) \nabla_{\perp}^{2} \psi+g \frac{\partial N}{\partial y}=\mu \nabla^{4} \psi, \\
& \left(\frac{\partial}{\partial t}+\hat{\mathbf{z}} \times \nabla \psi \cdot \nabla\right) N-\frac{2}{R_{0}} \frac{\partial \psi}{\partial y}=\chi \nabla_{\perp}^{2} N .
\end{aligned}
$$

Here $g=2 C_{s}^{2} / R_{0}$ is the effective centrifugal acceleration due to the nonuniform magnetic field with $C_{S}$ the acoustic speed. The collision terms on the right-hand sides of these equations are given for a fully ionized plasma. The kinematic viscosity is given in terms of the ion-ion collision frequency $\nu_{i i}$ by $\mu=(3 / 10) \rho_{c i}^{2} \nu_{i i}$, where $\rho_{c i}=\sqrt{T_{i} m_{i}} /(e B)$ is the ion gyro radius and $\nu_{i i}$ is the ion-ion collision frequency. The collisional diffusion coefficient $\chi$ depends on the electron-ion collision frequency $\nu_{e i}$ and can be written in the form $\chi=\left(1+T_{i} / T_{e}\right) \rho_{c e}^{2} \nu_{e i}$, where $\rho_{c e}=\sqrt{T_{e} m_{e}} /(e B)$ is the electron 
gyro radius. The diffusion terms have slightly different forms in a weakly ionized plasma dominated by collisions with neutrals. Equations (11) model particle density and potential structures that have no wave-vector component parallel to the magnetic field, so-called flute modes. This implies azimuthal symmetry, and we can employ a two-dimensional rectangular coordinate system in the $(R, Z)$ plane at any given $\Phi$. In order to conform with the notation used for the RB convection model, we have introduced $x$ as the radial coordinate and $y$ as the axial coordinate of the solenoid.

Using the collision model for fully ionized plasmas given in Eqs. (11), the structure of the RB model and the plasma convection model are very similar. Yet, there are some differences that are related to the fact that while $\theta$ in the former model represents the deviation from a linear background profile, $N=\ln n$ in the plasma model represents the logarithm of the full density, that is, it includes the equilibrium profile. We thus expand the particle density into a background and a fluctuating part according to $n(x, y, t) \rightarrow n_{0}(x)$ $+n(x, y, t)$, and assume for simplicity that there is no background potential profile and thus no equilibrium flows. Note that we have assumed that there is no dependence of the fluctuating quantities along the magnetic field. This is based on theoretical as well as experimental evidence indicating that flute perturbations dominate over drift waves on the bad curvature side of a nonuniformly magnetized plasma in those cases in which flute modes are not topologically forbidden due to magnetic rotational transform. ${ }^{15,16}$ Under these assumptions, we have

$$
N=\ln \left(n_{0}+n\right)=\ln n_{0}+\ln \left(1+n / n_{0}\right) \rightarrow N_{0}(x)+N(x, y, t) .
$$

Postulating a linear $N_{0}$ profile, corresponding to an exponential $n_{0}$ profile, $N_{0}(x)=N_{0}(d)+(d-x) / L_{n}$, Eq. (11b) reduces to

$$
\left(\frac{\partial}{\partial t}+\hat{\mathbf{z}} \times \nabla \psi \cdot \nabla\right) N+\left(\frac{1}{L_{n}}-\frac{2}{R_{0}}\right) \frac{\partial \psi}{\partial y}=\chi \nabla_{\perp}^{2} N .
$$

In analogy with Eq. (2), we introduce the dimensionless variables

$$
t \rightarrow \frac{\chi}{d^{2}} t, \quad \mathbf{x} \rightarrow \frac{\mathbf{x}}{d}, \quad \psi \rightarrow \frac{\psi}{\chi}, \quad N \rightarrow \frac{N}{\kappa-\zeta},
$$

where we have introduced the particle density gradient and field curvature parameters

$$
\kappa=-\frac{\partial \ln n_{0}}{\partial x}=\frac{d}{L_{n}}=N_{0}(0)-N_{0}(d), \quad \zeta=\frac{2 d}{R_{0}} .
$$

Equations (11) then take the dimensionless form

$$
\begin{aligned}
& \left(\frac{\partial}{\partial t}+\hat{\mathbf{z}} \times \nabla \psi \cdot \nabla\right) \nabla_{\perp}^{2} \psi+\operatorname{ScGr} \frac{\partial N}{\partial y}=\operatorname{Sc}_{\perp}^{4} \psi, \\
& \left(\frac{\partial}{\partial t}+\hat{\mathbf{z}} \times \nabla \psi \cdot \nabla\right) N+\frac{\partial \psi}{\partial y}=\nabla_{\perp}^{2} N,
\end{aligned}
$$

with the Schmidt number Sc denoting the ratio of the viscosity and particle diffusivity and Gr describing the effective buoyancy relative to collisional dissipation,

$$
\mathrm{Sc}=\frac{\mu}{\chi}, \quad \mathrm{Gr}=\frac{(\kappa-\zeta) g d^{3}}{\mu \chi} .
$$

Equations (16) are formally equivalent to the RB model by the substitutions

$$
N \rightarrow \theta, \quad \mathrm{Sc} \rightarrow \mathrm{Pr}, \quad \mathrm{Gr} \rightarrow \mathrm{Ra} .
$$

This means that the Lorenz model can be derived for the plasma convection problem under the same boundary conditions and truncations as described in Sec. II. From the stability analysis of the Lorenz equations presented in Sec. II, we found that the threshold for instability is given by $\mathrm{Gr}>\mathrm{Ra}_{c}$ $=27 \pi^{4} / 4$, which gives a threshold condition on the particle density gradient

$$
\kappa>\kappa_{c}=\zeta+\frac{27 \pi^{4}}{4 g d^{3}} \mu \chi .
$$

As mentioned in Sec. III, this threshold is finite, $\kappa_{c}=\zeta$, in the limit of vanishing dissipation. ${ }^{12-14}$

\section{THE DIFFUSIONLESS LORENZ MODEL}

A particular feature of the RB model for thermal convection is the nonconvective boundary conditions. In the thermal convection problem, the heat transport through the upper and lower boundaries is purely conductive, and in the isothermal version of the plasma convection problem the particle transport through these boundaries is exclusively due to collisional cross-field ambipolar diffusion governed by the electron collision frequency. This means that the transport through the layer is still limited by collisional diffusion through the boundaries, and we demonstrated at the end of Sec. II that under the Lorenz truncation the particle flux cannot exceed three times the diffusive flux in equilibrium.

This diffusion-limited flux is certainly not the situation typically encountered in atmospheric or ocean circulation, nor in magnetically confined laboratory or space plasmas. In these situations, it is generally recognized that convection and turbulence lead to strongly enhanced transport, which in many cases are orders of magnitude larger than the collisional transport. In the real world, the external drive is often not given by some fixed boundary conditions, but rather as an imposed flux due to a certain given rate of local heat or plasma production. The limitations of the nonconvective boundary conditions become particularly apparent in the limit of vanishing collisional diffusion $\chi$. In a flux-driven system, the background particle density or temperature gradient then has to grow beyond limits to accommodate the imposed flux. This means that the Prandtl and Rayleigh numbers both grow beyond limits, but the ratios

$$
\frac{\mathrm{Ra}}{\operatorname{Pr}^{2}}=\frac{d^{3}}{\mu^{3}} \alpha g \chi \Delta T, \quad \frac{\mathrm{Gr}}{\mathrm{Sc}^{2}}=\frac{d^{3}}{\mu^{3}} g \chi(\kappa-\zeta)
$$

remain finite if the heat flux, proportional to $\chi \Delta T$, or plasma flux, proportional to $\chi \kappa$, is kept constant. This is what is called the diffusionless limit of the Lorenz equations. ${ }^{17,18} \mathrm{Al}-$ though this limit is well posed mathematically, it does not 
make much physical sense for the RB boundary value problem due to the infinite background gradients required to drive a finite flux by diffusion through the boundaries.

In the next section, we shall present an alternative formulation of the Lorenz model that does not imply infinite gradients in the diffusionless limit, and which may correspond more to physical reality in the case of open boundaries. But before we embark on that, it may be useful to reformulate the Lorenz equations in a way that is more suitable for going to the diffusionless limit. By a simple change of variables

$$
t \rightarrow \sigma t, \quad \mathcal{U}=\mathcal{X} / \sigma, \quad \mathcal{V}=-\mathcal{Y} / \sigma, \quad \mathcal{W}=(r-\mathcal{Z}) / \sigma,
$$

time is normalized by the viscous time $d^{2} / \mu$ rather than the diverging diffusion time $d^{2} / \chi$, and the Lorenz system (5) takes the form

$$
\begin{aligned}
& \dot{\mathcal{U}}=-\mathcal{U}-\mathcal{V}, \\
& \dot{\mathcal{V}}=-\epsilon \mathcal{V}-\mathcal{W} \mathcal{U}, \\
& \dot{\mathcal{W}}=-\xi \mathcal{W}+\mathcal{U N}+F,
\end{aligned}
$$

where

$$
\epsilon=\frac{1}{\sigma}, \quad \xi=b \epsilon, \quad F=\frac{b r}{\sigma^{2}}=\epsilon \xi r .
$$

Since in the diffusionless limit $\sigma \rightarrow \infty$ and $r \rightarrow \infty$, while $F$ remains finite, the diffusionless Lorenz equations is the system (22) with $\epsilon=\xi=0$,

$$
\begin{aligned}
& \dot{\mathcal{U}}=-\mathcal{U}-\mathcal{V}, \\
& \dot{\mathcal{V}}=-\mathcal{W} \mathcal{U}, \\
& \dot{\mathcal{V}}=\mathcal{U} \mathcal{V}+F .
\end{aligned}
$$

The properties of this model will be described in some detail in Sec. VI.

In experiments in partially and fully ionized plasmas, we typically have $\sigma \sim 10-100$, so we may be tempted to neglect the contribution from electron collisions in the collision terms in Eqs. (11), which corresponds to the diffusionless limit. When the diffusion is small but finite, we consider a situation for which $\xi \sim \epsilon \ll 1$ but $r \gg 1$, hence the diffusive equilibrium $\mathcal{X}=\mathcal{Y}=\mathcal{Z}=0$, which corresponds to $\mathcal{U}=\mathcal{V}=0$ and $\mathcal{W}=F / \xi$, is unstable. In physical terms, this means that the temperature or particle density gradient is way above the dissipative instability threshold, and that the phase-space trajectory oscillates around the fixed points corresponding to the two convective states $\mathcal{X}=\mathcal{Y}= \pm \sqrt{b(r-1)}$ and $\mathcal{Z}=r-1$, which correspond to $\mathcal{U}=-\mathcal{V}= \pm \sqrt{F-\epsilon \xi}$ and $\mathcal{W}=\epsilon$. It may be instructive to relate the variable $\mathcal{W}$ directly to physical quantities, for instance to the modification of the temperature profile in the RB convection model. It is easily shown that this profile in dimensional units can be written

$$
\langle T(x, y, t)\rangle_{y}=T_{0}(x)+\frac{\Delta T}{\pi}\left(\frac{\mathcal{W}}{r \epsilon}-1\right) \sin \left(\frac{2 \pi x}{d}\right),
$$

where $T_{0}(x)=T_{0}(0)-(x / d) \Delta T$ is the linear equilibrium profile, which follows from Eq. (25) for the fixed point of the basic state given by $\mathcal{W}=F / \xi=r \epsilon$. In the conductive equilibrium, $\mathcal{W}=F / \xi=r \epsilon$ and hence $\langle T(x)\rangle_{y}=T_{0}(x)$ as expected. In the steady convective state, $\mathcal{W}=\epsilon$, and hence for $r \rightarrow \infty$ we have the temperature profile

$$
\begin{aligned}
\langle T\rangle_{y} & \rightarrow T_{0}(x)-\frac{\Delta T}{\pi} \sin \left(\frac{2 \pi x}{d}\right) \\
& =T_{0}(0)-\frac{\Delta T}{d}\left[x+\frac{d}{\pi} \sin \left(\frac{2 \pi x}{d}\right)\right] .
\end{aligned}
$$

As discussed in Sec. II, this gives the conductive flux $\Gamma=-\left.\chi \partial_{x} T\right|_{x=0}=3 \chi \Delta T / d$ through the boundary. In Sec. II we mentioned that for $r \gg 1$ we generally have that $\mathcal{Z} \sim r$. More precisely, $Z$ makes nonlinear periodic or chaotic oscillations around the fixed point $Z=r-1$, and we may write $\mathcal{Z}=\overline{\mathcal{Z}}$ $+\delta \mathcal{Z}$, where $\overline{\mathcal{Z}}=r-1$ is the time-averaged value of $\mathcal{Z}$ and $\delta \mathcal{Z}$ is the oscillating part with standard deviation much less than $r$. Thus a generalization of Eq. (26) to include the dynamic solutions of the Lorenz model is

$$
\langle T\rangle_{y} \rightarrow T_{0}(0)-\frac{\Delta T}{d}\left[x+\frac{d}{\pi}\left(1+\frac{\delta \mathcal{Z}}{r}\right) \sin \left(\frac{2 \pi x}{d}\right)\right] .
$$

This shows that the oscillation in the flux is of order $\delta \mathcal{Z} / r$ $\ll 1$ compared to the time-averaged flux, and that the timeaveraged flux is given by Eq. (26). The important implications of these observations are that in the diffusionless limit the periodic or chaotic oscillations are of little physical significance, since the profile and flux are only weakly perturbed by these oscillations. Moreover, the modification of the linear profile by the action of the convection cells is moderate and only enhances the diffusive flux by a factor less than 3. For this reason, sustainment of a finite flux in the diffusionless limit requires an infinite particle density or temperature gradient, which means that this truncation for thermal convection is not appropriate for description of strongly enhanced transport caused by gradient-driven fluid instabilities. This observation motivates the formulation of a model that does not limit the transport through the boundaries to be diffusive. This is the subject of the next section.

\section{LORENZ MODEL FOR PLASMA STREAMER STRUCTURES}

In this section, we will present an alternative formulation of the Lorenz model for plasma convection based on streamer-like flow structures described by Eqs. (11). These equations admit exact solutions in the form

$$
\begin{aligned}
& \psi(x, y, t)=\Psi(t) \sin k y, \\
& N(x, y, t)=\Theta(t) \cos k y,
\end{aligned}
$$

which do not satisfy the nonconvective boundary conditions at the radial positions $x=0$ and $x=d$ as imposed in the conventional approach. The convection cells of that approach 
have been replaced by radially elongated streamers that transport plasma in the radial direction from some source region to the left to some sink region to the right. The evolution of the streamer amplitudes is given by

$$
\begin{aligned}
& \dot{\Psi}=-\mu k^{2} \Psi-\frac{g}{k} \Theta, \\
& \dot{\Theta}=-\chi k^{2} \Theta-\frac{k}{d} \Psi \Pi,
\end{aligned}
$$

where we have introduced $\Pi=\kappa-\zeta$. Recall that these equations are based on the assumption of a stiff, exponential density profile of the form $n_{0}(x)=n_{d} \exp \left[(d-x) / L_{n}\right]$, where $\kappa=d / L_{n}$. As long as $n_{0}(x)$ is assumed time-independent, Eqs. (29) are linear, and a trivial stability analysis yields that the fixed point $\Psi=\Theta=0$ is unstable if

$$
\kappa>\zeta+\frac{K^{4}}{g d^{3}} \mu \chi
$$

where $K=k d$ is the dimensionless wave number introduced in Sec. II. If we compare this with the corresponding result for the most unstable convective cells, for which the aspect ratio is given by $K=\pi / \sqrt{2}$, we find that the dissipative contribution to the instability threshold, described by the second term on the right of inequalities (19) and (30) for the convection cells, exceeds the threshold for streamer structures by a factor 27 . This is solely due to the neglect of boundaries in the present case.

In the RB convection problem, the linear system corresponding to Eqs. (29) arises from neglecting the profile correction term, namely the second term on the right-hand side in Eq. (4b). This implies setting $\mathcal{Z}=0$ in Eq. (5c), making Eqs. (5a) and (5b) a linear system in $\mathcal{X}$ and $\mathcal{Y}$. In the convective streamer problem, the corresponding approximation appears through assuming a time-independent value of the parameter П. A nonlinear saturation of the instability can be achieved by allowing for a back-reaction of the streamers on the profile scale length through a time dependence of $\Pi(t)$ $=\kappa(t)-\zeta$, rather than as the profile correction of the form $\mathcal{C}(t) \sin (2 \pi x / d)$ used in the RB convection model. This ansatz has some support in experiments where there is clear evidence of resilient exponential density profiles. ${ }^{15,16}$ A plausible explanation of this feature is that the profile is close to the threshold for flute interchange instability because the unstable streamer structures give rise to radial plasma transport, which in turn flattens the density profile and brings the gradient back below the instability threshold. Opposing this tendency is the plasma production in the core plasma, so the resulting dynamics may be some sort of nonlinear oscillations around the threshold profile. The $y$-averaged radial particle flux density at radial position $x$ caused by the streamer filaments is given by

$$
\begin{aligned}
\Gamma(x) & =\left\langle n v_{x}\right\rangle_{y} \approx-n_{0}(x) \frac{\langle N \partial \phi / \partial y\rangle_{y}}{B} \\
& =-\frac{k}{2} n_{0}(x)\left(1+\frac{x}{R_{0}}\right) \Psi \Theta,
\end{aligned}
$$

where we have used that $R=R_{0}+x, B^{-1}=R /\left(B_{0} R_{0}\right)=B_{0}^{-1}$ $\left(1+x / R_{0}\right), \psi=\phi / B_{0}$, and $\left\langle\cos ^{2} k y\right\rangle_{y}=1 / 2$. In the case of an exponential density profile $n_{0}(x)=n_{d} \exp \left[(d-x) / L_{n}\right]$, the divergence of the flux density is computed to be

$$
\begin{aligned}
\frac{1}{R} \frac{\partial}{\partial R}(R \Gamma) & \approx-\frac{k}{2 R_{0}\left(1+x / R_{0}\right)} \frac{\partial}{\partial x}\left[\left(1+\frac{x}{R_{0}}\right)^{2} n_{0}(x)\right] \Psi \Theta \\
& \approx \frac{k n_{d}}{2}\left(\frac{1}{L_{n}}-\frac{2}{R_{0}}\right) \Psi \Theta .
\end{aligned}
$$

This shows that near the nondissipative instability threshold given by $\kappa=\zeta$ (which implies that $1 / L_{n}=2 / R_{0}$ ), the $y$-averaged convective flux is nearly solenoidal, implying that the streamer structures as described above will provide a particle flux through the layer without local accumulation of plasma that will distort the exponential shape of the density profile.

Let us now consider the particle number $\mathcal{N}$ inside a slab of unit lengths in the $Z$ and $\Phi$ directions and with thickness $d$ centered around $R_{0}$ in the radial direction. Assume that the plasma source produces a number $\Gamma_{s}$ of free electrons per unit area and time that enter the slab from the left, and that plasma escapes by a combination of convective transport and collisional diffusion at the right at $x=d$. The global particle conservation equation then takes the form

$$
\dot{\mathcal{N}}=-\Gamma-\Gamma_{\chi}+\Sigma=\frac{k}{2} n_{d} \Psi \Theta+\frac{2 n_{d}}{R_{0}}\left(S-D_{\chi}\right),
$$

where $\Gamma_{\chi}=-\chi \partial n_{0} / \partial x=2 \chi n_{d} / R_{0}$ is the classical diffusive flux and $S=2 \Sigma /\left(R_{0} n_{d}\right)$ is the effective diffusion coefficient that would be required to provide the flux $\Sigma$, both in a background gradient assumed to be close to the nondissipative threshold, $L_{n}=R_{0} / 2$. Let us also assume that the density profile maintains the exponential form, but we allow the dimensionless inverse scale length $\kappa=d / L_{n}$ to vary with time. The number of particles in the slab can then be expressed as

$$
\mathcal{N}(t)=n_{d} d \int_{0}^{d} d x \exp \left[\kappa\left(1-\frac{x}{d}\right)\right]=\frac{n_{d} d}{\kappa}(\exp \kappa-1) .
$$

Proximity to the ideal threshold and the thin slab approximation $2 d / R_{0} \ll 1$ allows us to expand the exponential to second order in $\kappa$, which yields $\dot{\mathcal{N}} \approx d n_{d} \dot{\Pi} / 2$, and hence Eq. (33) reduces to

$$
\dot{\Pi}=\frac{k}{d} \Psi \Theta-\frac{2 \chi}{d^{2}} \Pi+\frac{2 \zeta}{d^{2}}(S-\chi) .
$$

By introducing the viscous time scale and the following change of variables: 


$$
t \rightarrow \mu k^{2} t, \quad \mathcal{U}=\frac{1}{k d \mu} \Psi, \quad \mathcal{V}=\frac{g}{k^{4} d \mu^{2}} \Theta, \quad \mathcal{W}=\frac{g}{k^{4} d \mu^{2}} \Pi,
$$

Eqs. (29) and (35) reduce to the Lorenz equations in the form of Eqs. (5), where

$$
\epsilon=\frac{1}{\sigma}=\frac{\chi}{\mu}, \quad \xi=\frac{2}{K^{2}} \epsilon, \quad F=\frac{2}{K^{6}} \frac{d^{3}}{\mu^{3}} g \zeta(S-\chi),
$$

and $K=k d$. It is interesting to compare Eq. (37) with the corresponding expressions following from the thermal convection model. These can be obtained from Eqs. (7), (20), and (23) and yield

$$
\begin{aligned}
& \epsilon=\frac{1}{\sigma}=\frac{\chi}{\mu}, \quad \xi=\frac{4 \pi^{2}}{\pi^{2}+K^{2}} \epsilon, \\
& F=\frac{4 \pi^{2}}{\left(\pi^{2}+K^{2}\right)^{4}} \frac{d^{3}}{\mu^{3}} g \zeta \chi\left(\frac{\kappa}{\zeta}-1\right) .
\end{aligned}
$$

For $K \sim \pi$, the geometrical factors depending on $k d$ in Eq. (38) are of the same order of magnitude as the corresponding factors in Eq. (37). Hence, the dissipation rates $\epsilon$ and $\xi$ are comparable for the convective streamer and convective cell models for identical plasma parameters. However, the forcing terms $F$ are comparable in the two models only if $\kappa / \zeta \sim S / \chi$. This means that in the diffusionless limit $(\chi \rightarrow 0$, $F$ finite) we must have that $\kappa / \zeta \rightarrow \infty$, an observation made already in Sec. IV. An implication of the discussion in that section is that the thermal convection model does not allow effective diffusion coefficients exceeding $3 \chi$, and hence infinitely large gradients are required to drive a finite flux in the diffusionless limit.

It is well known that for the Lorenz model the phasespace trajectory constitutes periodic or chaotic oscillations around one or both of the fixed points $\mathcal{U}=-\mathcal{V}= \pm \sqrt{F-\epsilon \xi}$, $\mathcal{W}=\xi$. In Sec. VI, it will be demonstrated that in the diffusionless limit $(\epsilon, \xi \rightarrow 0)$, and for strong forcing $(F \gtrsim 1)$ we have that the amplitudes of these oscillations scale as $\delta \mathcal{U} \sim \delta \mathcal{V} \sim \delta \mathcal{W} \sim F$. From Eqs. (36) and (37), we then have that

$$
\delta \mathcal{W}=\frac{g d^{3}}{K^{4} \mu^{2}} \delta \Pi \sim F=\frac{2}{K^{6}} \frac{d^{3}}{\mu^{3}} g \zeta(S-\chi) .
$$

In the diffusionless limit, we have the time averages $\overline{\mathcal{W}}=\bar{\Pi}$ $=0$, and hence $\bar{\kappa}=\zeta$. Thus, since $\delta \kappa=\delta \Pi$, we find from Eq. (39) the following estimate for the relative fluctuation level of the density gradient,

$$
\frac{\delta \kappa}{\bar{\kappa}} \sim \frac{2}{K^{2}} \frac{S}{\mu}=\frac{2 \tau_{\mu}}{\tau_{s}} .
$$

Here we have introduced the viscous relaxation time $\tau_{\mu}=\left(k^{2} \mu\right)^{-1}$ for the streamer structures in the direction perpendicular to the density gradient, and the relaxation time $\tau_{s}=d^{2} / S$ along the density gradient determined by the convective transport through the layer. This shows that the oscillations of the profile gradient around the threshold gradient are small if $\tau_{\mu} \ll \tau_{s}$. Thus, increased viscosity $\mu$ leads to smaller profile oscillations, but also to smaller streamer amplitudes, since from Eqs. (36) and (37) and $\delta \mathcal{U} \sim \delta \mathcal{V} \sim F$ $\sim \mu^{-3}$ we have that $\delta \Psi \propto \mu^{-2}$ and $\delta \Theta \propto \mu^{-1}$. Since the flux due to the streamers is proportional to the time-average $\overline{\Psi \Theta}$, a constant flux can be maintained as $\mu$ increases only if the phase difference between $\Psi$ and $\Theta$ changes to increase the time average of the product. How this comes about will appear through the numerical solutions described in the next section.

\section{BASIC FEATURES OF THE DLE AND LE}

The Lorenz equations are perhaps the most famous model of nonlinear dynamics and has been established as a paradigm for dissipative chaos. The diffusionless Lorenz equations are much less known. This model was first proposed by Sprott in 1994 in a search for the simplest possible chaotic three-dimensional dynamical systems, ${ }^{17}$ and later studied in some mathematical detail as a diffusionless limit of the Lorenz equations. ${ }^{18}$ Here we present a numerical study of some basic properties that can be of relevance for detectable characteristics for plasma systems that might be described by the model, and refer the reader to Ref. 18 for a more exhaustive mathematical and numerical treatment.

For a constant profile gradient $W$, Eqs. (22a) and (22b) represent a linear stability problem with solutions of the form $\mathcal{X}, \mathcal{Y} \sim \exp (\lambda t)$, with characteristic equation $\lambda^{2}+\lambda-\mathcal{W}=0$, and roots $\lambda=(-1 \pm \sqrt{4 \mathcal{W}+1}) / 2$. This yields exponential growth of the convective structures for $W>0$ and damping for $W<0$. The ensuing structures give rise to an anomalous plasma flux down the pressure gradient given by $\Gamma=\mathcal{U N}$. This plasma loss is compensated by a plasma source term $F$ as expressed by Eq. (22c).

Invoking also Eq. (22c) for the profile, the system has two stationary solutions or fixed points, $( \pm \sqrt{\mathcal{W}}, \mp \sqrt{\mathcal{W}}, 0)$. These two solutions are physically equivalent, since the system exhibits the symmetry $(\mathcal{U}, \mathcal{V}, \mathcal{W}) \rightarrow(-\mathcal{U},-\mathcal{V}, \mathcal{W})$. For finite diffusion, that is, for the full Lorenz model, this symmetry is broken. The fixed points represent solutions where the profile gradient is at the instability threshold, coexisting with a streamer with amplitude sufficient to provide anomalous flux that exactly balances the plasma production rate. There also exists an important one-parameter class of solutions $\mathcal{U}=\mathcal{V}=0, \mathcal{W}=\mathcal{W}_{0}+F t$, which describes the unlimited linear growth of the profile gradient driven by the plasma source when no streamers are excited to provide anomalous transport.

Introducing $\mathbf{X}=(\mathcal{U}, \mathcal{V}, \mathcal{W})$ and letting $\mathbf{X}^{ \pm}$denote the fixed points, we can perform a linear stability analysis of the fixed points by introducing perturbations of the form $\mathbf{X}-\mathbf{X}^{ \pm}$ $=\delta \mathbf{X}_{j}^{ \pm} \exp \lambda_{j} t, \quad j=1,2,3$. The characteristic equation is $\lambda_{j}^{3}+\lambda_{j}^{2}+\lambda_{j} F+2 F=0$. For $F>0$ there is one negative eigenvalue $\lambda_{1}=\mu$ and two complex eigenvalues with positive real part; $\lambda_{2,3}=\sigma \pm i \omega$. This implies that for $F>0$, the fixed points are unstable and are classified as spiral saddle points of index 2 (Ref. 19). Chaos results from a competition between the attracting and the repelling properties of the fixed points, as can be illustrated by plotting their numerically computed 


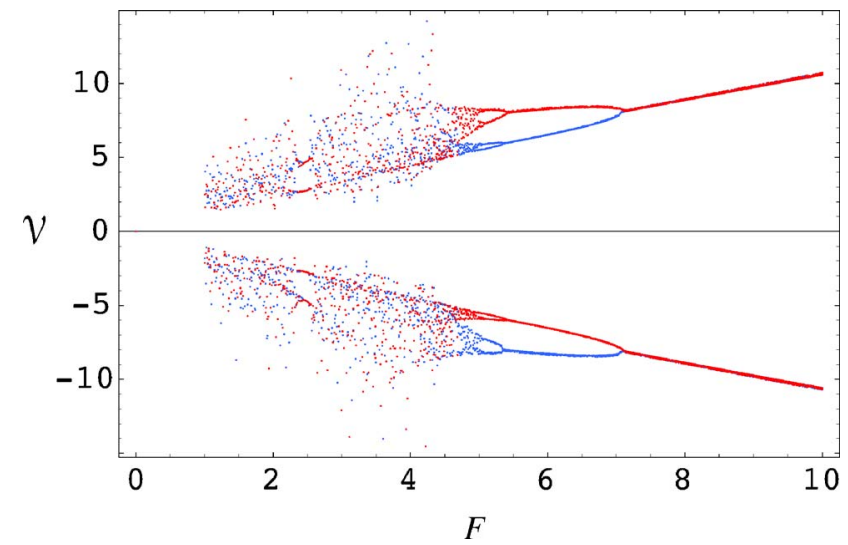

FIG. 1. (Color online) Bifurcation diagram for $F=1 \rightarrow 10$ (red) and $F=10 \rightarrow 1$ (blue).

phase-space trajectories or by their corresponding bifurcation diagrams.

Figure 1 shows a bifurcation diagram for strong drive $F \in(1,10)$. In the plot, the value of $\mathcal{V}$ is marked by a dot each time the phase-space trajectory crosses the $\mathcal{U}$ - $\mathcal{V}$ plane in the direction from positive to negative $\mathcal{W}$. The blue points derive from starting the computation of the trajectory for $F=10$ and with a more or less randomly chosen initial condition and starting to plot the points when the trajectory has converged to the attractor. Then the value of $F$ is reduced by $\Delta F=0.001$ and a new trajectory is computed with the end point of the previous one as initial condition, and intersection values of $\mathcal{V}$ with the $\mathcal{U}-\mathcal{V}$ plane are plotted. This procedure is continued for 1000 values of $F$ until $F=1$. The red points are obtained by starting at $F=1$ and increasing $F$ in the same steps until $F=10$. The red points are plotted on top of the blue ones, so in those cases where the red and the blue points belong to the same limit cycle, only the red ones appear in the figure. This is the case for the lines appearing in the plot for $F>7$ (we pay no attention to accurate computation of bifurcation values of $F$ in this paper). In those cases in which overlapping red and blue points bifurcate into one red and one blue branch the corresponding trajectories have converged to different phase-space attractors. An example is the bifurcation near $F=7$, where the symmetric limit cycle appearing for larger $F$ splits into two asymmetric limit cycles in a symmetry-breaking (pitchfork) bifurcation. This means that the initial conditions for the red and blue trajectories in those cases belong to the basin of attraction for different attractors. Obviously, the branch chosen by the computation when $F$ approaches the bifurcation point depends on where the trajectory is just prior to the bifurcation, and hence is sensitive to the initial point chosen for the computation. Thus, one should keep in mind that this bifurcation plot only serves to illustrate the existence of different attractors for the same value of $F$, and does not exclude the existence of other attractors with different basins of attraction.

Figure 2 illustrates the phase-space orbit for a stable limit cycle $(F=7.5)$ and a weakly chaotic orbit $(F=4.5)$. In both cases, the orbits intersect the $\mathcal{U}-\mathcal{V}$ plane from positive to negative $\mathcal{W}$ twice during one cycle (quasicycle in the chaotic case), which implies that the same orbit has points in the
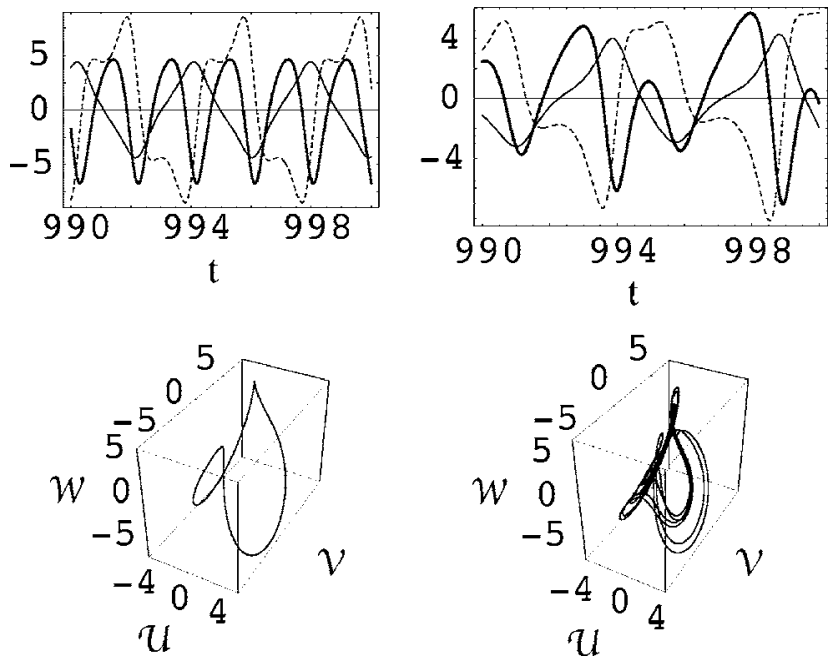

FIG. 2. Upper left: $\mathcal{U}(t)$ (dotted), $\mathcal{V}(t)$ (full thin), $\mathcal{W}(t)$ (full thick) for $F=7.5$. Lower left: Phase-space orbit for $F=7.5$. Upper right: $\mathcal{U}(t)$ (dotted), $\mathcal{V}(t)$ (full thin), $\mathcal{W}(t)$ (full thick) for $F=4.5$. Lower right: Phase-space orbit for $F=4.5$.

bifurcation diagram both at positive and negative $\mathcal{V}$. Hence, for a given value of $F$, the red branches on both sides of the $F$ axis belong to the same orbit, and the blue branches to another orbit. The pitchfork bifurcation that occurs at $F \approx 7$ is a splitting of the limit cycle observed at the lower left in Fig. 2 into two different limit cycle orbits corresponding to the red and the blue branches in the bifurcation diagram, respectively. However, the cascade of bifurcations starting near $F=5$ represents period doublings of the same limit cycle. A sequence of new period doubling bifurcations then takes place as $F$ is reduced until a transition to chaos occurs.

Figure 3 shows the corresponding bifurcation diagram for $F \in(0.1,1)$. Most of this regime is chaotic, with small windows of limit cycle behavior that undergo period doubling routes to chaos as $F$ is increased. A chaotic orbit is shown to the left in Fig. $4(F=0.9)$, and a periodic orbit that is just beyond a period doubling bifurcation $(F=0.3)$ is shown to the right in the same figure.

For weaker drive, orbits tend to be attracted to the solution $\mathbf{X}=\left(0,0, \mathcal{W}_{0}+F t\right)$, and the basin of attraction to

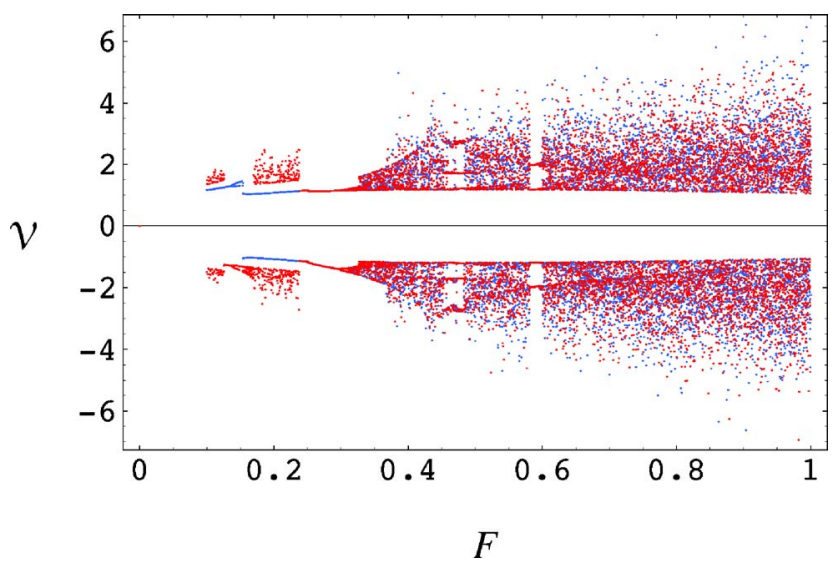

FIG. 3. (Color online) Bifurcation diagram for $F=0.1 \rightarrow 1$ (red) and $F=1 \rightarrow 0.1$ (blue). 

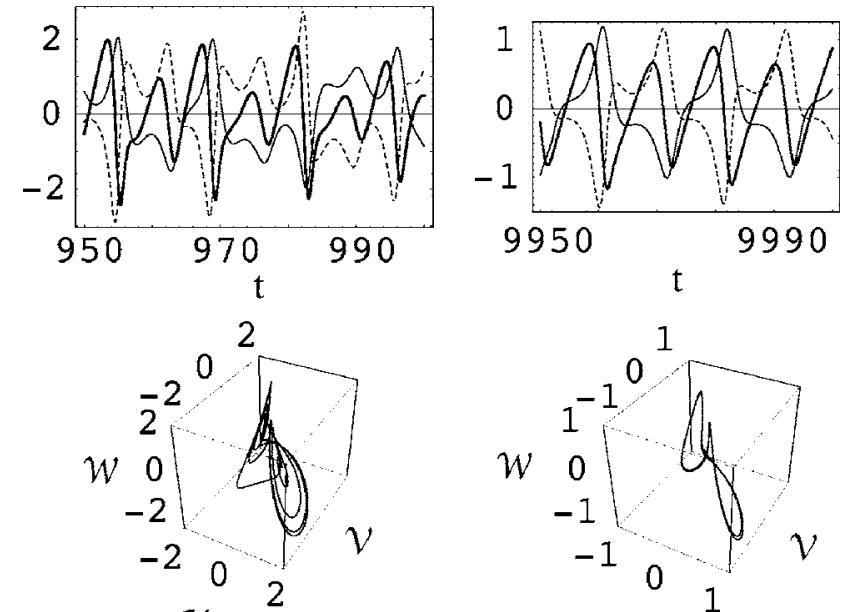

U

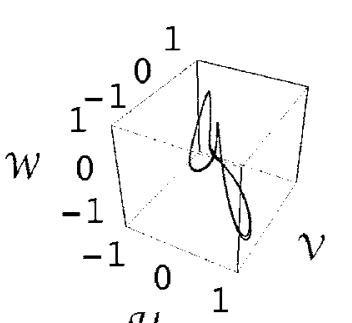

u

FIG. 4. Upper left: $\mathcal{U}(t)$ (dotted), $\mathcal{V}(t)$ (full thin), $\mathcal{W}(t)$ (full thick) for $F=0.9$. Lower left: Phase-space orbit for $F=0.9$. Upper right: $\mathcal{U}(t)$ (dotted), $\mathcal{V}(t)$ (full thin), $\mathcal{W}(t)$ (full thick) for $F=0.3$. Lower right: Phase-space orbit for $F=0.3$.

bounded orbits becomes very small. This basin grows considerably with introduction of weak diffusion in the equations. A bifurcation diagram with $\epsilon=\xi=0.001$ is shown in Fig. 5. This return to the full Lorenz system does not give a noticeable change in the structure of the plot for orbits that start sufficiently close to the bounded attractor, but makes it easier to produce the plot since the basin of attraction is enlarged by the introduction of diffusion. In Fig. 6, we plot the orbit corresponding to the red points in Fig. 5 for $F=0.1$ and 0.01 . The bifurcation diagrams show that the orbit is chaotic, which would also be apparent from the phasespace plot if it were plotted for much longer time than the three cycles shown in the orbit plot. Examination of the time traces of $\mathcal{U}, \mathcal{V}$, and $\mathcal{W}$ shows that the polarity of the peaks in $\mathcal{U}$ and $\mathcal{V}$ shifts in a random manner from cycle to cycle. There is also a weak chaotic variation in the amplitude of each peak, but the main chaotic ingredient is the random choice the orbit makes between encircling either the fixed point $\mathbf{X}^{-}$or the point $\mathbf{X}^{+}$. The left and the right lobe of the attractor shown in the lower part of Fig. 6 are geometrically identical, although it is not apparent from the figure due to

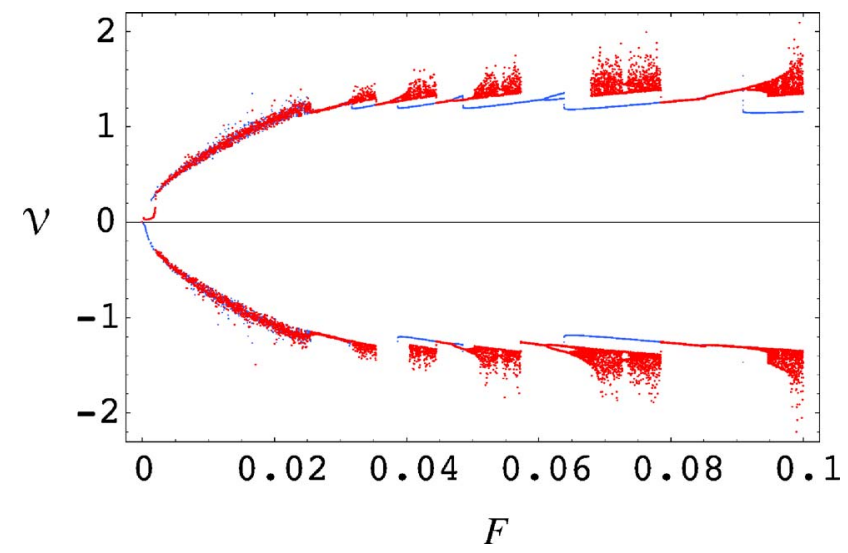

FIG. 5. (Color online) Bifurcation diagram for $F=0.0001 \rightarrow 0.1$ (red) and $F=0.1 \rightarrow 0.0001$ (blue).
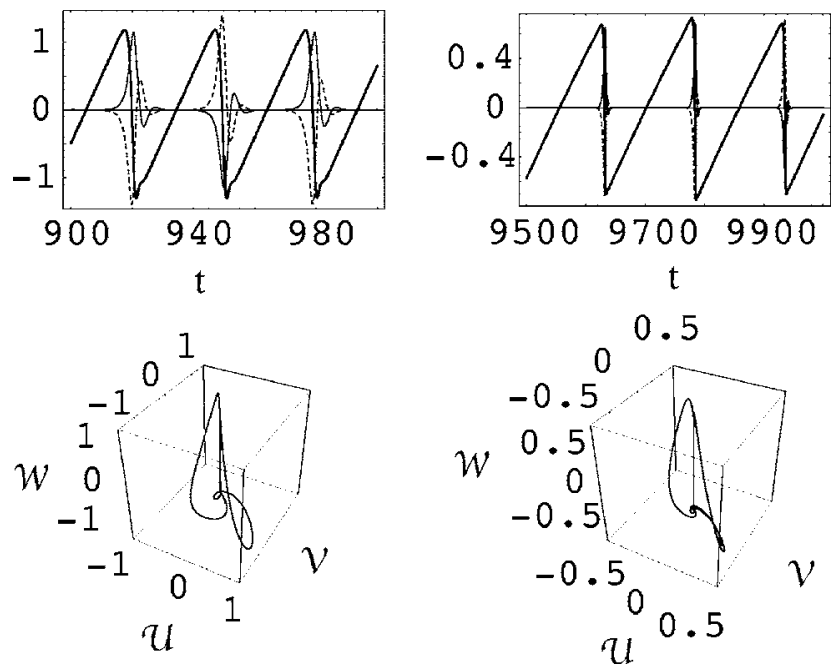

FIG. 6. Upper left: $\mathcal{U}(t)$ (dotted), $\mathcal{V}(t)$ (full thin), $\mathcal{W}(t)$ (full thick) for $F=0.1$. Lower left: Phase-space orbit for $F=0.1$. Upper right: $\mathcal{U}(t)$ (dotted), $\mathcal{V}(t)$ (full thin), $\mathcal{W}(t)$ (full thick) for $F=0.01$. Lower right: Phase-space orbit for $F=0.01$.

the angle of view (a clearer view is provided in the upper left of Fig. 9). The vertical straight part of the orbit corresponds to the part of the time trace when $\mathcal{U} \approx \mathcal{V} \approx 0$ and $\mathcal{W}$ grows linearly. In physical terms, the profile gradient grows linearly and the streamer is suppressed during this phase of the cycle. At a certain threshold of the gradient, the streamer grows rapidly with polarity either +1 or -1 (or alternatively, with phase 0 or $\pi$ ). This leads to a burst of convective transport and a corresponding fall in the gradient and decay of the streamer amplitude.

The random nature of the "flips" in polarity is demonstrated in Fig. 7. The sawtooth trace of $\mathcal{W}(t)$ allows us to define the times $t_{n}, n=1,2,3, \ldots$ when the orbit intersects the $\mathcal{U}-\mathcal{V}$ plane from positive to negative $\mathcal{W}(t)$. These are the times when the streamer is bursting, and we record the polarity or sign of $\mathcal{U}$, that is, $x_{n}=\operatorname{sgn}\left[\mathcal{U}\left(t_{n}\right)\right]$. The time series $\left\{x_{n}\right\}$ for $F=0.01$ and $n=1, \ldots, 200$ is plotted at the upper left of Fig. 7, and on the upper right the time series of the cumulative sum $y_{n}=\sum_{m=1}^{n} x_{m}$ is shown. If the flips $\left\{x_{n}\right\}$ constitute a
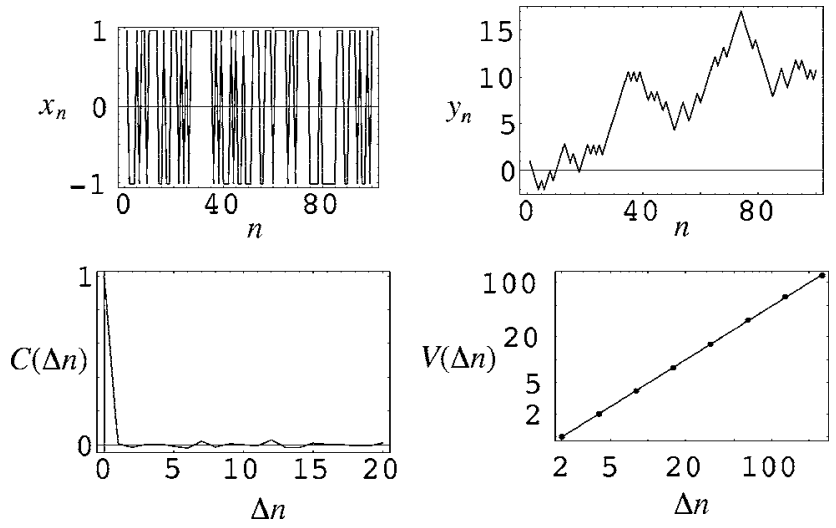

FIG. 7. Upper left: Time series $x(n)=\operatorname{sgn}\left[\mathcal{U}\left(t_{n}\right)\right], n=1,2,3, \ldots$ at every crossing $t_{n}$ of orbit from positive to negative $\mathcal{W}$. Upper right: Cumulative sum $y(n)=\sum_{m=1}^{n} x(n)$. Lower left: Autocovariance of time series $x(n)$. Lower right: Variogram of time series $y(n)$. 

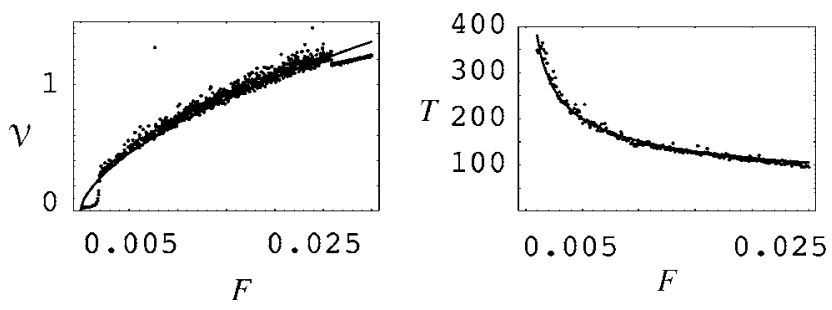

FIG. 8. Left: Dots are the positive part of the Poincaré plot for $F \in(0.0001,0.025)$ and full curve is a plot of the function $11 F^{0.6}$. Right: Dots are period between sawteeth for $\mathcal{W}(t)$ vs $F$ and full curve is a plot of the function $24 F^{-0.4}$.

random process without memory, the process $\left\{y_{n}\right\}$ is a Brownian random walk. The autocovariance function $C(\Delta n)$ for $\left\{x_{n}\right\}$ estimated for a time series of $N=4096$ points is shown on the lower left, and indicates that there is no memory in the process. A more robust method to exclude long-range memory is estimation of the variogram $V(\Delta n)=\sum_{n=1}^{N-\Delta n}\left(y_{n+\Delta n}-y_{n}\right)^{2}$. To the lower right in Fig. 7 we present a double logarithmic plot of the variogram. The linear fit to this curve has slope $2 H$, where $H=0.499$. Thus, within the accuracy of this estimate we have $V(\Delta n)$ $=c(\Delta n)^{0.5}$, which is expected for a Brownian random walk, and the numerical solution of the weakly forced DLE works perfectly well as a random coin flipper.

The bifurcation diagram also shows that other types of attractors exist in this range of $F$, including stable limit cycles around one fixed point (as shown, for instance, by the blue branch for $F \approx 0.1$ ), and such limit cycles undergoing period-doubling bifurcations. For $F<0.025$ the orbits look like those shown on the right in Fig. 6. They are sawtoothlike in the profile gradient time trace, and the streamer amplitudes display bursts that grow shorter with decreasing $F$. The amplitude variation of the streamer with $F$ can be fitted well by the curve $\mathcal{V}=11 F^{0.6}$, as shown on the left in Fig. 8 . The streamer amplitude follows the amplitude of the profile sawtooth, and hence the period between bursts should go as $T \sim \mathcal{V}^{-1} \sim F^{-0.4}$. To the right in Fig. 8 we have plotted $T$ versus $F$ as estimated from the numerically computed orbits, together with the curve $T=24 F^{-0.4}$.

\section{THE PERILS OF DIMENSION REDUCTION}

Toward the end of Sec. II we pointed out that the conventional reduction of the RB partial differential equations to the Lorenz equations through the Galerkin procedure for convective cells puts a strong limitation on the energy flux when diffusion is small. Numerical solutions to the RB equations show that this limitation does not exist for the full boundary value problem, since the flux is carried through the boundaries by diffusion through strong local gradients near the boundary. Such strong gradients are incompatible with the Galerkin truncation. The reduction of the phase-space dimension by this truncation is therefore unable to capture the real physics for the strongly driven convective cell problem in the weak diffusion limit, but a similar reduction may prevail as a valid description for the streamer problem when boundaries are open.
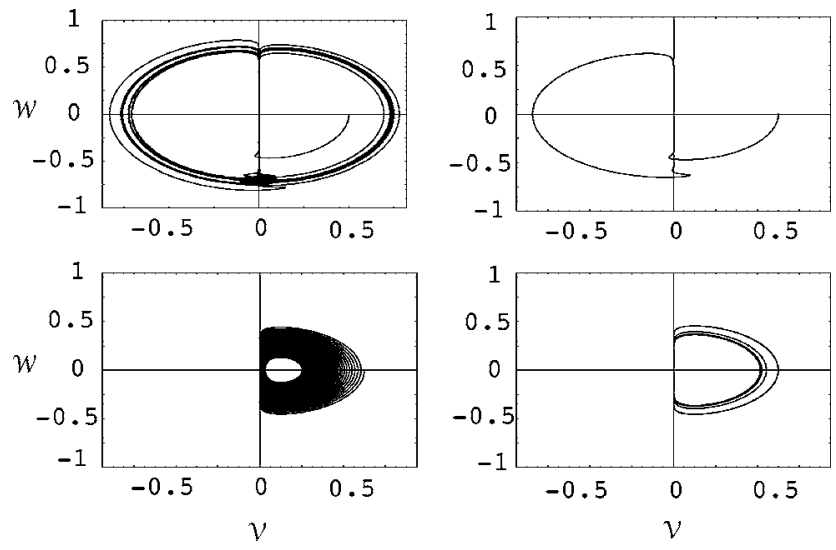

FIG. 9. Upper left: Transient orbit of LE projected on the $\mathcal{V}-\mathcal{W}$ plane. Upper right: Transient orbit of DLE. Lower left: Transient orbit for slaved model with diffusion. Lower right: Segments of transient orbit for diffusionless slaved model.

The reduction of the LE to the DLE did not reduce the phase-space dimension, but did reduce the number of free parameters from three to one, the number of fixed points from three to two, and introduced a reflexive symmetry in the problem. Introduction of a small diffusion does not seem to have a qualitative impact on the attractors traced through the numerical solutions in this paper, but it seems to influence the basin of attraction for these attractors for weak forcing $F<0.1$. For such weak forcing, the numerical solutions display periodic bursts in $\mathcal{U}$ and $\mathcal{V}$ for which $\mathcal{U} \approx-\mathcal{V}$ (see Fig. 6). This observation suggest a dimension reduction by invoking the slaving principle $\mathcal{U}=-\mathcal{V}$, which reduces the Lorenz equations (22) to

$$
\begin{aligned}
& \dot{\mathcal{V}}=-\epsilon \mathcal{V}+\mathcal{W} \mathcal{V}, \\
& \dot{\mathcal{W}}=-\xi \mathcal{W}-\mathcal{V}^{2}+F .
\end{aligned}
$$

A similar model has previously been suggested as a paradigm for bursting and large-scale intermittency due to backreaction by turbulent convection on the pressure profile. ${ }^{20}$ Even though the approximation made by invoking the slaving principle is always a very accurate one for $F<0.1$, the reduction of dimension from three to two has the qualitative consequence of removing chaos (chaos does not exist in phase-space flows of dimension less than three). The three fixed points of the LE (and the two fixed points of the DLE) prevail, but the two unstable spiral saddles become stable spiral foci for the slaved LE and neutrally stable centers for the slaved DLE. Some consequences of these changes can be observed in Fig. 9, where we have made some plots of orbits projected on the $\mathcal{V}-\mathcal{W}$ plane for LE, DLE, and the slaved model for $F=0.01$ and initial state $\mathbf{X}_{0}=(-0.5,0.5,0)$. On the upper left of this figure is plotted the transient orbit $t \in(0,3000)$ of the LE with diffusion rate $\epsilon=\xi=0.001$. The orbit quickly ends up on a chaotic attractor where it flips randomly between the left and the right lobe shown in the figure. On the upper right is shown the transient orbit $t \in(0,300)$ of DLE. After one turn in the left lobe, the orbit is attracted to the unbounded solution $\mathbf{X}=\left(0,0, \mathcal{W}_{0}+F t\right)$. On 
the lower left we have the transient orbit $t \in(0,3000)$ for the slaved LE with diffusion rate $\epsilon=\xi=0.001$, demonstrating that the point $(0,0, \sqrt{F})$ is a stable spiral focus in this model. When $\epsilon=\xi=0$, the resulting slaved DLE exhibit orbits that are very slowly attracted toward the fixed point, reflecting the neutrally stable nature of this fixed point. On the lower right figure, the outer lobe corresponds to the orbit for $t \in(0,1000)$, and the lobes further in to $t \in(100000,101000)$, $t \in(200000,201000), t \in(300000,301000)$, and $t \in(400000$, $401000)$, respectively. This demonstrates that the attraction of the orbit becomes slower the closer it comes to the fixed point, and that it takes the character of an almost perfectly periodic nonlinear oscillation. This example illustrates that approximations that reduce the dimensionality of a model should be handled with great caution, since it can cause qualitative changes in the structure of the solutions.

\section{DISCUSSION AND CONCLUSIONS}

The possibility of low-dimensional chaotic transport dynamics in a magnetized laboratory plasma was suggested by Rypdal and Ratynskaia in connection with the description of experiments in the Helimak configuration. ${ }^{16}$ The Helimak is the simplest toroidal plasma configuration that exhibits an equilibrium without the presence of turbulent stress, and is very suitable for studies of low-frequency gradient driven instabilities and their routes to chaos and turbulence in the presence of magnetic-field curvature, and related to this, cross-field convective transport and the formation of resilient plasma profiles. The models developed here, however, could be relevant for many other magnetized plasma systems subject to gradient driven instabilities and anomalous transport. We have shown in this paper that low-dimensional dynamical system models can self-consistently describe strong anomalous transport in terms of streamers with profile backreaction, even in the limit of strong forcing and vanishing diffusion.

The key to comparing the theory to a given experimental situation is to estimate the forcing parameter $F$ given by Eq. (37). This parameter depends strongly on the geometrical parameters, the plasma parameters, and the strength of the flux imposed by the forcing of the system. In particular, it depends very strongly on the ion viscosity $\left(F \sim \mu^{-3}\right)$, which means that a large range of values of $F$ can be expected for actual physical systems. Our numerical solutions, which are supported by analytical estimates, ${ }^{18}$ indicate that the solutions to the DLE are stable limit cycles for $F \gg 1$, but exhibit chaos for most values of $F \lesssim 1$.

In a real plasma system it is not likely that one will observe "clean" low-dimensional behavior. The typical situ- ation will be that high-dimensional turbulent dynamics dominate the smaller scales, and that the low-dimensional dynamics may govern the global scales of the system. Detection of low-dimensional dynamics should therefore focus on observables that are dominated by the global scales, and advanced data analysis techniques are required to extract the lowdimensional component in the signal, and compute Lyapunov exponents and attractor dimension, quantities that are easily computed from the numerical solutions of the LE and DLE. Such techniques have been applied, for instance, to fusion plasmas, ${ }^{21,22}$ where the search for low-dimensional attractors has so far been mostly negative. However, application to geomagnetic indices, which characterize the global state of the magnetosphere-ionosphere system, has produced evidence of chaotic low-dimensional behavior ${ }^{23}$ consistent with certain models for the global dynamics of this system. ${ }^{24}$ We are now performing such an analysis for the Helimak experiments, ${ }^{16}$ and we plan to publish the results in a separate paper.

\section{ACKNOWLEDGMENT}

This work was supported under project 171076/V30 of the Norwegian Research Council.

${ }^{1}$ Lord Rayleigh, Philos. Mag. 32, 529 (1916)

${ }^{2}$ E. N. Lorenz, J. Atmos. Sci. 20, 130 (1963).

${ }^{3}$ J. H. Curry, J. R. Herring, J. Loncaric, and S. A. Orzag, J. Fluid Mech. 147, 1 (1984).

${ }^{4}$ K. V. Roberts and J. B. Taylor, Phys. Fluids 8, 315 (1965).

${ }^{5}$ H. Sugama and M. Wakatani, J. Phys. Soc. Jpn. 59, 3937 (1990).

${ }^{6}$ O. Pogutse, W. Kerner, V. Gribkov, S. Bazdenkov, and M. Osipenko, Plasma Phys. Controlled Fusion 36, 1963 (1994).

${ }^{7}$ O. E. Garcia, N. H. Bian, J.-V. Paulsen, S. Benkadda, and K. Rypdal, Plasma Phys. Controlled Fusion 45, 919 (2003).

${ }^{8}$ O. E. Garcia and N. H. Bian, Phys. Rev. E 68, 047301 (2003).

${ }^{9}$ N. H. Bian and O. E. Garcia, Phys. Plasmas 12, 042307 (2005).

${ }^{10}$ O. E. Garcia, N. H. Bian, V. Naulin, A. H. Nielsen, and J. J. Rasmussen, Phys. Scr., T T122, 104 (2006).

${ }^{11}$ K. Rypdal, J.-V. Paulsen, O. E. Garcia, S. Ratynskaia, and V. Demidov, Nonlinear Processes Geophys. 10, 139 (2003).

${ }^{12}$ O. E. Garcia, J. Plasma Phys. 65, 81 (2001).

${ }^{13}$ O. E. Garcia, Eur. J. Phys. 24, 313 (2003).

${ }^{14}$ B. Lehnert, Phys. Fluids 5, 432 (1962).

${ }^{15}$ K. Rypdal and S. Ratynskaia, Phys. Plasmas 11, 4623 (2004).

${ }^{16}$ K. Rypdal and S. Ratynskaia, Phys. Rev. Lett. 94, 225002 (2005).

${ }^{17}$ J. C. Sprott, Phys. Rev. E 50, 647 (1994).

${ }^{18}$ G. van der Schrier and L. R. M. Maas, Physica D 141, 19 (2000).

${ }^{19}$ R. C. Hilborn, Chaos and Nonlinear Dynamics (Oxford University Press, Oxford, 2000)

${ }^{20}$ N. H. Bian and O. E. Garcia, Phys. Plasmas 10, 4696 (2003).

${ }^{21}$ F. Sattin and E. Martines, Phys. Plasmas 6, 100 (1999).

${ }^{22}$ V. Hynönen, O. Dumbrajs, A. W. Degeling, T. Kurki-Suonio, and H. Urano, Plasma Phys. Controlled Fusion 46, 1409 (2004).

${ }^{23}$ M. A. Athanasiu and G. P. Pavlos, Nonlinear Processes Geophys. 8, 95 (2001).

${ }^{24}$ W. Horton, R. S. Weigel, and J. C. Sprott, Phys. Plasmas 8, 2946 (2001). 